\title{
Minimum Viable User EXperience: A Framework for Supporting Product Design in Startups
}

\author{
Laura Hokkanen $^{(\bowtie)}$, Kati Kuusinen, and Kaisa Väänänen \\ Department of Pervasive Computing, Tampere University of Technology, \\ Korkeakoulunkatu 1, 33720 Tampere, Finland \\ \{Laura. Hokkanen, Kati.Kuusinen, Kaisa.Vaananen\}@tut.fi
}

\begin{abstract}
Startups operate with small resources in time pressure. Thus, building minimal product versions to test and validate ideas has emerged as a way to avoid wasteful creation of complicated products which may be proven unsuccessful in the markets. Often, design of these early product versions needs to be done fast and with little advance information from end-users. In this paper we introduce the Minimum Viable User eXperience (MVUX) that aims at providing users a good enough user experience already in the early, minimal versions of the product. MVUX enables communication of the envisioned product value, gathering of meaningful feedback, and it can promote positive word of mouth. To understand what MVUX consists of, we conducted an interview study with 17 entrepreneurs from 12 small startups. The main elements of MVUX recognized are Attractiveness, Approachability, Professionalism, and Selling the Idea. We present the structured framework and elements' contributing qualities.
\end{abstract}

\section{Introduction}

Global markets are being infiltrated by small startups with their innovative new products and business models. Software startups are characterized with scarce resources, little to none operating history, and time pressure [1]. One competitive advantage with startups compared to large organizations is their ability to move fast and adapt to changing circumstances [2]. However, as founding teams of startups often consist of only a few individuals, the team's skills are naturally limited. For the same reason, the primary business objective of startups is to survive [3]. To survive, startups need to make the most out of their limited resources. Customer development [4] and Lean startup method [5], that have been widely adopted and taught by accelerators and entrepreneurship programs [6], emphasize gathering fast feedback from customers, and testing product ideas with minimal product versions or Minimum Viable Product (MVP) as referred by Ries [5]. While Lean Startup has no scientific evidence for effectiveness in business creation, the method is influencing how entrepreneurs approach product development [6, 7].

While validating business potential with minimal product versions and real customers to minimize unnecessary risk, gathering useful feedback with early product 
versions can be challenging. One challenge is that insufficient or disturbing user experience (UX) might reduce the user feedback and make the users concentrate mainly on the appearance of the user interface [8]. At the worst, poor UX can lead the user only to criticize the UX even if the product idea itself was good. [8] Benefits of delivering good UX from the earliest product version can be positive word of mouth advertisement [9], and users using the product for longer.

The goal of this paper is to identify and structure the UX elements that are essential when building early product versions in small software startups. To understand the elements of desirable UX of early product versions, we introduce the concept and framework of Minimum Viable User eXperience that aims at providing UX that enables users to understand and gain value already from the early product versions. Correspondingly, startup is then able to collect more meaningful feedback from potential customers over a longer period of time since users do not abandon the product.

In this paper, we report results of a two-phase interview study we conducted in Finland. In the first phase we interviewed 13 entrepreneurs from eight startups. All the startups were building, or had recently built, first versions of their products. Based on the analysis of these interviews, we created the initial MVUX framework. The framework is based on the assumption that MVUX is realized in the software being under development when (1) user can perform the core use cases to gain value, (2) basic hygiene factors for usability and appearance are in place, and (3) the startup is able to get enough of feedback and data to validate and further develop the product idea. To evaluate the MVUX framework, we then interviewed four entrepreneurs of four more startups, all having expertise in UX. Through the interviews, we answer the following research questions: (1) what are the goals and key elements of MVUX from the startups' perspective and (2) how can MVUX design framework help startups at the early phases of their product and business development.

The rest of this paper is structured as follows. Section 2 presents related work on characteristics of software startups and their ways of working, and UX practices. Section 3 presents context and methods of our study. In Sect. 4 we present the results of our study including the UX elements considered important by startups, as well as the results of the evaluation of the MVUX framework. Section 5 discusses the results and Sect. 6 presents the conclusions for the paper.

\section{Related Work}

\subsection{Characteristics of Software Startups}

Engineering and business concerns in software startups are more extensive than in established companies [2]. Those concerns include having scarce resources, being young and immature, operating with novel technologies in dynamic markets. Software startups are also influenced by divergent stakeholders such as investors, customers, partners, and competitors. [2] Also, customer-focused approach seems to be more crucial for small companies [2]: When the customer is happy with the software, it literally means more work and increased business opportunities for the small company 
as the happy customer wants more and is willing to recommend the software to others [10]. Because of unestablished customer base, such positive word of mouth and keeping the existing customers satisfied is essential for startups.

The professionalism of the entrepreneurs themselves often acts as a primary information source for startups due to unestablished stakeholder networks and customer base [3]. Moreover, people factors tend to be even more crucial for startups than for larger companies in the success or failure of the software [2]. Thus, the entrepreneur team is in a key role in keeping the startup focused and moving ahead [2]. For startups, short time to market is one of the most critical process goals [2]. Since a fundamental goal of a process is to describe the way an organization develops its software in a predictable and repeatable fashion, benefits of an established process do not meet essential needs of software startups $[2,3]$. Therefore, startups require more informal and lightweight approaches.

New entrepreneurial practices Customer development [4] and Lean startup method [5] have been gaining attention in recent years. These practices emphasize that startups should concentrate on producing customer value and avoid wasteful activities, i.e. non-value adding activities. Although academic research on how well Customer development and the Lean startup method work is scarce, those methods have been widely adopted by incubators, accelerators and university entrepreneurship courses [6]. The Lean startup [5] suggests that by validating hypotheses of customer's problems startups find a problem/solution fit that indicates there is business potential in solving a specific problem with a particular solution. Once the problem/solution fit is established, the startup should validate what product suites to deliver the solution. For finding validation, startups should build minimum viable products (MVP) that are then tested with potential customers. An MVP should be built with as little resources as possible yet it needs to enable testing the current hypothesis. Furthermore, Ries [5] emphasizes that the key performance indicators need to be measured when "getting out of the building" with the MVPs. From these experiments, startup should gain validated learning [5]. This Build-Measure-Learn (BML) cycle should be continued until a product/market fit is found and startups should also be prepared to discard the MVPs if they do not measure up to validating sustainable business opportunity [5].

\subsection{User Experience Work}

$\mathrm{UX}$ is defined as "a person's perceptions and responses that result from the use or anticipated use of a product, system or service" [11]. Also, UX is often divided into practical-oriented and hedonic dimensions [12]. The first dimension includes aspects related to ease of use, productivity, and usability while the latter concentrates on users' emotions such as enjoyment and motivation. Regarding UX development in industry, companies in general tend to focus more on the practical qualities of UX while paying less attention to the hedonic ones [13].

UX design has roots in human-centered design (HCD) [11] that starts with thorough user research and design activities which are followed by design iterations. All in all, developing UX involves gaining understanding of the user and the context of use, designing and developing for good UX, and evaluating the resulting outcome [11]. 
While understanding users is considered important for startups [7], startups generally do not afford to follow rigorous methods for UX development. Research on UX development in startups is scarce. May [14] describes a case from applying lean methodology in a startup and recommends planning the UX activities in from early on. Klein [15] presents lightweight methods for UX work in lean startups. Finally, Hokkanen et al. [8] report that lack of UX expertise and time constrains hinder the startup from collecting useful feedback from users.

\section{Methods, Research Context, and Participants}

\subsection{Course of the Study}

To address our research goal of understanding which UX factors are essential when building early product versions in startups, semi-structured interviews were chosen as the data gathering method. The study was conducted in two phases. In the first phase we interviewed 13 entrepreneurs from 8 small startups in order to establish the MVUX framework. In the second phase, four entrepreneurs with UX expertise were interviewed to evaluate the created MVUX framework. Altogether, 12 interview sessions with 17 interviewees were conducted. All the interviews were conducted by one researcher and they lasted between 50-90 min. Interviews were audio recorded and transcribed for analysis. Participants were searched by going through Finnish startup incubator and accelerator programs. Some startups were recruited through directly contacting them based on their web page while others were recruited by advertising in the premises of one incubator program.

In the first phase, eight semi-structured interviews were conducted to understand the early design decisions and UX goals in startups. Initial results from these interviews, describing how startups start UX design, and what practices are beneficial at that stage, are reported in [7]. During the interviews, we introduced the general concept of MVUX to each interviewee. Participants were then asked to write down on a paper their goals and central elements for UX of their early product version intended to be deployed to users. Differences in UX goals between the earliest and complete product version were also shortly discussed. In all the interviews, focus was on UX related motivations and practices. However, activities such as product and business development were covered superficially to understand their impact on UX design.

In the second phase, four semi-structured interviews were conducted to evaluate the MVUX framework established based on the results of the first phase. The concept of MVUX was first discussed with the interviewee after which we presented them the initial MVUX framework. Then we asked questions about the interviewee's perception on the ability of the MVUX framework to cover the necessary UX elements without including unnecessary elements. In addition, we studied the usefulness of the framework by discussing with the interviewees how startups could utilize the MVUX framework while creating early product versions.

In both phases, analysis was done from the written transcripts utilizing iterative thematic coding. Main themes were established based on the interview questions while sub-themes emerged from the data. Terms the interviewees used to describe the goals 
and central elements of UX of the early product version were collected to construct the MVUX framework. Those terms were used as low-level elements on which the main elements of the framework were created using a bottom-up approach as follows. In total, 43 unique low-level elements were abstracted from the interview data. These low-level elements were divided into groups based on similarity to form mid-level elements of MVUX. Finally, mid-level elements were grouped based on similarity to determine the main elements of MVUX. In the grouping of elements both the term as well as the context in which the element was discussed was taken into consideration.

\subsection{Participants}

First Phase. Startups participating the first phase consisted of one to six person teams each creating one single software product (Table 1). In this paper, we number the startups from 'ST11' to 'ST18', to differentiate them from the startups that participated our previous study [8].

Table 1. Summary of startups and interviewees participating the first phase. Legend: $\mathrm{CEO}=$ Chief Executive Officer, $\mathrm{UXD}=$ User Experience Designer, $\mathrm{B} 2 \mathrm{~B}=$ Business to Business, B2C $=$ Business to Consumer, SaaS $=$ Software as a Service.

\begin{tabular}{|c|c|c|c|c|c|}
\hline Startup & Interviewees & $\begin{array}{l}\text { Company } \\
\text { established }\end{array}$ & $\begin{array}{l}\text { Size of } \\
\text { startup }\end{array}$ & Product & Market \\
\hline ST11 & H01 (CEO) & 2013 & 1 & Online marketplace & $\begin{array}{l}\mathrm{B} 2 \mathrm{~B}, \\
\mathrm{~B} 2 \mathrm{C}\end{array}$ \\
\hline ST12 & $\begin{array}{l}\text { H02 (CEO), } \\
\text { H03 }\end{array}$ & 2014 & 6 & Online marketplace & $\mathrm{B} 2 \mathrm{C}$ \\
\hline ST13 & H04 (UXD) & 2014 & 4 & $\begin{array}{l}\text { Online community and } \\
\text { marketplace }\end{array}$ & $\begin{array}{l}\mathrm{B} 2 \mathrm{~B}, \\
\mathrm{~B} 2 \mathrm{C}\end{array}$ \\
\hline ST14 & $\begin{array}{c}\mathrm{H} 05, \mathrm{H} 06 \\
\text { (CEO) }\end{array}$ & 2014 & 2 & SaaS for pet owners & $\mathrm{B} 2 \mathrm{C}$ \\
\hline ST15 & $\begin{array}{l}\text { H07 (CEO), } \\
\text { H08 }\end{array}$ & 2011 & 2 & Automation software & B2B \\
\hline ST16 & H09 (CEO) & 2014 & 5 & $\begin{array}{c}\text { Mobile sports } \\
\text { application }\end{array}$ & $\begin{array}{l}\mathrm{B} 2 \mathrm{~B}, \\
\mathrm{~B} 2 \mathrm{C}\end{array}$ \\
\hline ST17 & $\begin{array}{l}\text { H10, H11, } \\
\text { H12 }\end{array}$ & - & 3 & $\begin{array}{l}\text { Mobile personal } \\
\text { finances application }\end{array}$ & $\mathrm{B} 2 \mathrm{C}$ \\
\hline ST18 & H13 (UXD) & 2015 & 3 & $\begin{array}{l}\text { Mobile social } \\
\text { application }\end{array}$ & $\mathrm{B} 2 \mathrm{C}$ \\
\hline
\end{tabular}

Second Phase. In the second phase we interviewed four entrepreneurs of four other small startups to evaluate the MVUX framework created in the first phase (Table 2). $\mathrm{H} 15$ and $\mathrm{H} 16$ worked full time in startups, while $\mathrm{H} 14$ and $\mathrm{H} 17$ were employed also outside their startups. Interviewees H14, H15 and H16 worked as UX designers. H16 was the CEO of ST21, and worked also on product development. All the interviewees had been developing software products or services in startups. 
Table 2. Participants of the second phase interviews.

\begin{tabular}{l|l|l|l}
\hline Startup & Interviewee & $\begin{array}{l}\text { Experience in } \\
\text { entrepreneurship (Years) }\end{array}$ & Education \\
\hline ST19 & H14 & 3 & $\begin{array}{l}\text { Bachelor of Interactive } \\
\text { Technology }\end{array}$ \\
\hline ST20 & H15 & 3 & Bachelor of Arts and Media \\
\hline ST21 & H16 & 3 & PhD, Interactive Technology \\
\hline ST22 & H17 & 2 & $\begin{array}{l}\text { Master of Science student, } \\
\text { majoring in UX }\end{array}$ \\
\hline
\end{tabular}

\section{Findings}

\subsection{Elements of MVUX}

Those startups participating in the first phase were creating or had recently created limited versions of their product. UX goals of these product versions varied among startups depending on what they sought to achieve with the product version. Table 3 presents the hierarchical categorization of low-level elements mentioned by interviewees and then grouped to form mid-level elements, and how mid-level elemenst were further grouped to form the main elements of MVUX.

The most common goal was that the product UX should be intuitive to use (with six low-level elements). Furthermore, it was considered necessary to create a UI that was simple (5) and easy to use (5) to enable smooth start for the user. For the B2B case of ST15, in which the acceptance of end-users was important for convincing the pilot customer, H07 commented: "The product had to be so easy to use that everyone would agree to start using it. That was the first requirement." [H07] There was more diversity in how startups wanted the user to experience the product: humane (4), visual (5) or having a feel of novelty (3). Depending on the origin of the product idea, the early version of the product could also be built to fulfill the entrepreneur's needs. H06 from ST14 explained that their first version was developed to serve their own interests: "We thought technical looking graphs would be cool and bring a sense of high-tech. [...] Then we realized normal people don't want to see that. You should have like soft hightech. The high-tech Apple has, and not like laser beams." [H06]

Hooking, or making the user to stay and want to come back was mentioned three times as well. These were related to needs to gain data that proved interest in the product, or showed how users behaved with the UI. Goals related to the product being functioning or technically working were mentioned three times. Depending on the product idea, communicating that the solution and application was credible (4) or efficient (3) was considered important by some startups (ST11, ST14, ST17) while for others it did not matter. For example, in the case of mobile personal finances application (ST17), it was crucial the product would be perceived as something the user can trust from early on. 
Table 3. Elements of MVUX

\begin{tabular}{|c|c|c|}
\hline Main element & Mid-level element & Low-level element \\
\hline \multirow[t]{15}{*}{ Attractive } & \multirow[t]{5}{*}{ Visual (5) } & Visual (ST14) \\
\hline & & Visual experience (ST16) \\
\hline & & Good visual appearance (ST11) \\
\hline & & Modern visual appearance (ST13) \\
\hline & & Not technical looking (ST14) \\
\hline & \multirow[t]{5}{*}{ Humane (5) } & Likable enough (ST12) \\
\hline & & Storytelling (ST13) \\
\hline & & Personal (ST17) \\
\hline & & Easy to approach (ST14) \\
\hline & & Cozy and warm (ST14) \\
\hline & \multirow[t]{3}{*}{ Novel (3) } & Fresh (ST12) \\
\hline & & $\begin{array}{l}\text { Differentiation from regular services } \\
\text { (ST13) }\end{array}$ \\
\hline & & Strong colours to differentiate (ST11) \\
\hline & \multirow{2}{*}{ Hooking (3) } & Gamification (ST18) \\
\hline & & Hooking (ST13, ST18) \\
\hline \multirow[t]{11}{*}{ Approachable } & \multirow[t]{6}{*}{ Intuitive (6) } & Familiar UI elements (ST13) \\
\hline & & Familiarity (ST14) \\
\hline & & Intuitive (ST17) \\
\hline & & No learning curve (ST18) \\
\hline & & Understandable (ST18) \\
\hline & & Explicit (ST16) \\
\hline & \multirow[t]{2}{*}{ Easy (5) } & Easy to browse products (ST13) \\
\hline & & Easy to use (ST12, ST15, ST16, ST18) \\
\hline & \multirow[t]{3}{*}{ Simple (5) } & Simple (ST12, ST14, ST15) \\
\hline & & Simple design (ST11) \\
\hline & & Minimal design (ST11) \\
\hline \multirow[t]{10}{*}{ Professional } & \multirow[t]{4}{*}{ Credible (4) } & Premium (ST17) \\
\hline & & Reliable (ST11) \\
\hline & & Secure (ST17) \\
\hline & & Credible (ST11) \\
\hline & \multirow[t]{3}{*}{ Functioning (3) } & Functioning (ST15) \\
\hline & & Smooth (ST17) \\
\hline & & Device independence (ST14) \\
\hline & \multirow[t]{3}{*}{ Efficient (3) } & Compact (ST14) \\
\hline & & Fast (ST17) \\
\hline & & See by glancing (ST14) \\
\hline \multirow{3}{*}{$\begin{array}{l}\text { Selling the } \\
\text { Idea }\end{array}$} & \multirow[t]{3}{*}{ Introducing the idea (5) } & First impression (ST17) \\
\hline & & Introducing the idea (ST11) \\
\hline & & Example pictures (ST11) \\
\hline
\end{tabular}


Table 3. (Continued)

\begin{tabular}{l|l|l}
\hline Main element & Mid-level element & Low-level element \\
\hline \multirow{4}{*}{\begin{tabular}{c} 
Building brand \& fan base \\
\cline { 2 - 3 }
\end{tabular}} & Lobbing (ST15) \\
\cline { 3 - 3 } & & Solution (ST12) \\
\cline { 3 - 3 } & & Traction (ST12) \\
\cline { 3 - 3 } & Exciting (ST12) \\
\cline { 2 - 3 } & Social (ST17) \\
\hline
\end{tabular}

Being able to introduce the product idea and show the value in it was one of the mid-level elements abstracted from the low-level elements. Goals considering brand creation and getting fans for the product included four low-level elements. In case of ST11, starting to create positive word of mouth influenced how the UX was designed. H02 told that he would like users to see the product as exciting so that they would tell their friends about it.

\subsection{MVUX Framework}

The elements four main elements of MVUX are Attractiveness, Approachability, Professionalism and Selling the Idea. Classification of mid-level elements into these categories is demonstrated in Fig. 1. At the bottom of the Fig. 1 is Selling the Idea which is the main aim of MVUX since it offers the startup a possibility to get feedback from users who actually understand the product idea. The three other main elements

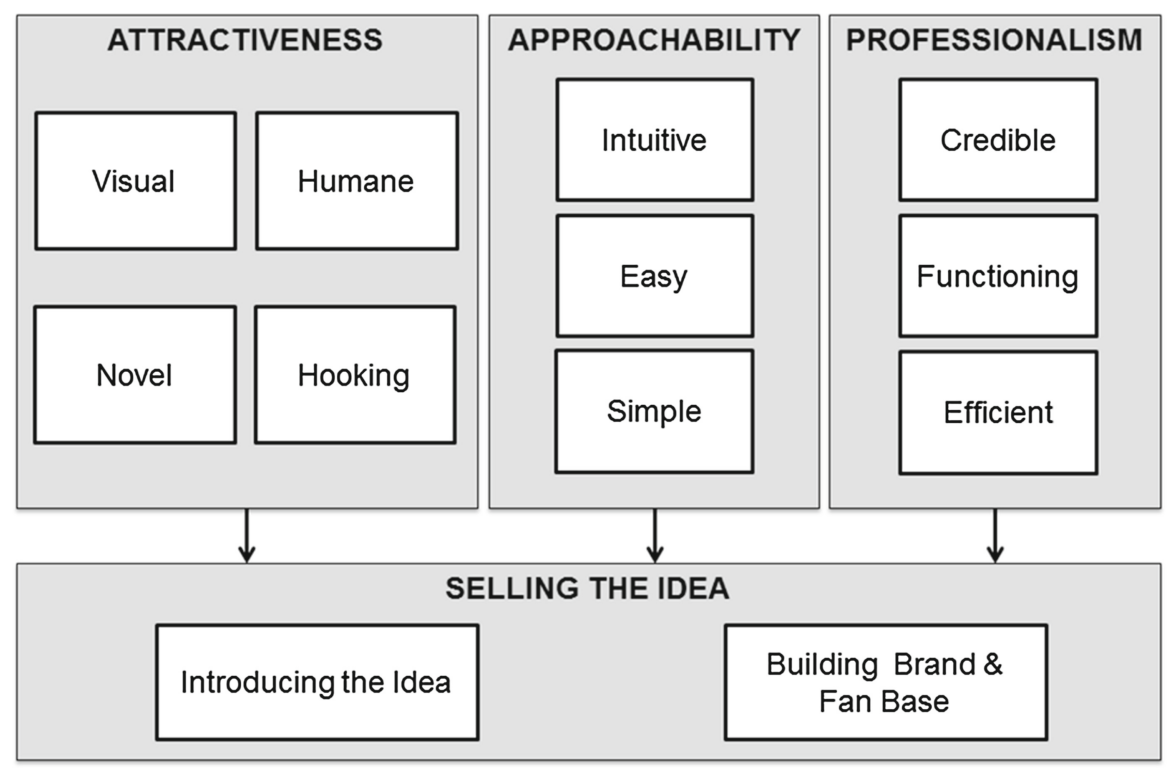

Fig. 1. MVUX framework for supporting early product development in startups. 
(Attractiveness, Approachability, and Professionalism) create the foundation for the user to be interested in the product and to start using it. These three elements can also be seen affecting the user in different phases of getting to know the product. The first impression of the product is influenced by making the early product version attractive. With approachable elements, the usage is made easy and comfortable. Giving a professional image of the product, and the startup, is the result of a well-functioning, efficient product.

\subsection{Validation of the MVUX Framework}

Impressions on the MVUX Framework. Interviewees considered that the elements of the framework cover well the needs for UX in an early product version. H16 thought that having a framework to guide developing UX for new products in startups would be very useful. The importance of different elements was discussed with the interviewees. According to H14, the element Selling the Idea communicated that the attributes enabling to sell the product need to be taken into consideration also in UX design. In contrast, H15 felt that selling the product can be done by marketing it and thus it does not require having good UX or even the product itself in the beginning- even though building the planned product might then be too difficult for the startup team (H15). Optimization of internet marketing can help in introducing the idea and creating a (fan) community (H15).

Being able to communicate the value proposition of the company was mentioned by H14 as a critical part of the early phases of their startup, and this was mainly done with text on web pages. H16 mentioned that various means are required to convince different stakeholder groups since buyers and users can be in very different positions. However, in addition to being able to evoke buyers' interest, the importance of users accepting the new product was brought up by H14, H15 and H16. Attractiveness and Approachability were considered as important parts of an early product version. However, H15 commented that having too polished visual design can create false expectations for the completeness of the product. For Professionalism, H15 and H16 both thought that it can be achieved - and is strongly affected by - other functions of the company such as marketing or personal contacts to companies in B2B markets, or in the case of $\mathrm{B} 2 \mathrm{C}$ market by who recommends the product to the user.

Usefulness of the MVUX Framework. The possibility to use MVUX framework when building the early product versions was discussed with the interviewees. H15, H16 and H17 said that startups could benefit from using a framework to remind themselves of where to focus in UX. However, the importance of each element depends on the product that is used. Also, the meaning and perception of each element is subjective. Furthermore, measuring users' perception of the product is necessary for understanding whether the intended UX was achieved in the product. While all interviewees regarded talking with users as the most valuable asset in creating good UX, H16 also stated that they could imagine using the framework to evaluate if the UX is good enough. Evaluation could be done by the startup team or with users by lightweight methods. To support the use of framework, H14, H16 and H17 thought that 
practical advice and examples would be needed to design graphical elements that support the wanted UX. However, graphical style was seen as something that can be easily created with existing tools for UI development as well as by utilizing image banks (H14, H15). H16 wished that the MVUX framework should indicate the iterative nature of creating products in startups. Also H14 and H15 mentioned iterative process starting form early releases - to be essential for successful product development in startups.

\section{Research Validity}

Since our study was qualitative, we assess our research quality in terms of credibility, transferability, dependability, and conclusions confirmability [16].

Credibility. We identified no major threats to credibility. Since the participants themselves wrote down the elements they considered essential for the UX of early versions, the study is less prone to interpretation error. However, we did not discuss the MVUX framework with participants of the first phase to evaluate interpretation issues.

Regarding the transferability of the results to other contexts, our study was conducted with 12 small Finnish software startups. We consider that our descriptive findings are transferable to similar startups. However, as startups - to a certain degree reflect the entrepreneurs themselves; personal characteristics may reduce the transferability of the results. In addition, when transferring the MVUX framework to other contexts, product type and the user must be considered. Transferability of the MVUX framework should be further analyzed with other startups.

Threats to dependability include that the studied startups did not form a random sample, instead convenience sampling was utilized. However, we utilized open sampling method in which new participants are recruited after interviewing the previous one to increase variation in the sample. Despite concentrating on Finnish startups, our study increases richness of related research that has been conducted, for instance, in Ireland [3] and in Ecuador [10].

Finally, threats to confirmability include that a single researcher planned, conducted and analyzed the study. The researcher, however, reflected with other researchers in every phase of the study. Finally, the MVUX framework was audited in a group of three researchers.

\section{Discussion}

Our contribution is in proposing a framework of UX elements that are essential to the early product versions startups create. Considering that the related research on startups in general and especially on their UX work is very limited, our study offers new insight both for the academia and for startups. In startups, the elements of MVUX could be used to guide the UX design of early product versions. Especially in the early phases, startups benefit from lightweight methods - such as promoted by [14] - and could also use MVUX framework to support the design decisions. However, further research 
should be done to understand and validate how MVUX can be used to support startups' UX strategy. Our initial validation shows that using MVUX framework with lightweight tools for implementing graphics design, and for measuring the perceived experience, would be beneficial in creating early product versions.

The goals and key qualities for UX of the early product versions had recurring themes from which we abstracted the elements of MVUX. Startups had different goals for what they wanted to achieve with their early product versions [7], and, accordingly, goals for UX varied. As reported in [7], startups also had different amounts of acquired understanding of their target users as well as previous validation of the product idea. This provided a wide scale of goals and qualities that reflected the different situations the startups were in. The four main elements of MVUX that we found are Attractiveness, Approachability, Professionalism, and Selling the Idea.

Based on our evaluation of MVUX framework with startup representatives that had expertise in UX, the MVUX framework covers the most important elements of UX in the early stages of startup's product development. However, the level of importance of different elements varies in products. Additionally, comparing the elements to our assumptions in the beginning of the study we can see how they are connected. We assumed that to communicate the product idea and UX well enough, the user should be able to perform the core use cases that answer to user's needs. Furthermore, we estimated the UX in these use cases should be at a satisfying level that does not disturb the user. These are in line with the elements Approachability and Professionalism that aim to provide trouble-free UX that shows the user that the product is trustworthy. Our third assumption for MVUX was that it needs to enable startup to gain feedback and data for validation and further development. This would be achieved through elements of Selling the Idea and Attractiveness. The element Attractiveness has a role in getting users interested in the product as well as hooking them to keep using the product. Selling the Idea part needs to be in place to raise interest in users, to communicate the product idea clearly, and to show how the product creates value to user so they will keep using the product. Implementation of elements of Attractiveness and Selling the Idea enables continuous data collection from longer usage as well as users being able to give feedback on the product idea while having no confusion on what the product is about. However, our initial assumptions did not emphasize the attractiveness and good visual design of the product, while the results of this study show that they are considered important in startups.

These results serve to create understanding of how UX should be taken into consideration when startups create their early product versions that are used by real user. Our study consisted of 12 Finnish-based companies so companies' motivations and goals are influenced by the Finnish business and startup culture. Furthermore, the end-users' preferred design elements may be influenced by the culture. Further research is needed to validate how well the discovered elements suit to the needs of startups and end-users in general. 


\section{Conclusions}

In this paper we introduced the results of our two-phase interview study of 17 entrepreneurs from 12 startups. We presented the framework of Minimum Viable User eXperience (MVUX) that represents ways in which UX can be focused on already in early product versions. To gain value from building early product versions, MVUX enables the startup to collect meaningful feedback and data for validating and further developing the product idea. We abstracted the elements of MVUX through a bottom-up analysis of startups' goals and key elements for UX of early product versions. From these elements, a framework for supporting UX design in early product development was established. In the second phase of the study, the constructed framework was evaluated with experts of both entrepreneurship and UX. As a conclusion, we present the MVUX framework where the main elements of MVUX were defined as Attractiveness, Approachability, Professionalism and Selling the Idea.

Open Access. This chapter is distributed under the terms of the Creative Commons Attribution-NonCommercial 4.0 International License (http://creativecommons.org/licenses/bync/4.0/), which permits any noncommercial use, duplication, adaptation, distribution and reproduction in any medium or format, as long as you give appropriate credit to the original author(s) and the source, a link is provided to the Creative Commons license and any changes made are indicated.

The images or other third party material in this chapter are included in the work's Creative Commons license, unless indicated otherwise in the credit line; if such material is not included in the work's Creative Commons license and the respective action is not permitted by statutory regulation, users will need to obtain permission from the license holder to duplicate, adapt or reproduce the material.

\section{References}

1. Paternoster, N., Giardino, C., Unterkalmsteiner, M., et al.: Software development in startup companies: a systematic mapping study. Inf. Softw. Technol. 56(10), 1200-1218 (2014)

2. Sutton, S.M.: The role of process in a software start-up. IEEE Softw. 17(4), 33-39 (2000)

3. Coleman, G., O'Connor, R.: An investigation into software development process formation in software start-ups. J. Enterp. Inf. 21(6), 633-648 (2008)

4. Blank, S.: Why the lean start-up changes everything. Harv. Bus. Rev. 91, 63-72 (2013)

5. Ries, E.: The Lean Startup: How Today's Entrepreneurs Use Continuous Innovation to Create Radically Successful Businesses. Random House LLC, New York (2011)

6. York, J.L., Danes, J.E.: Customer development, innovation, and decision-making biases in the lean startup. J. Small Bus. Strategy 24(2), 21-39 (2014)

7. Hokkanen, L., Kuusinen, K., Väänänen, K.: Early product design in startups: towards a UX strategy. In: Abrahamsson, P., et al. (eds.) PROFES 2015. LNCS, vol. 9459, pp. 217-224. Springer, Heidelberg (2015). doi:10.1007/978-3-319-26844-6_16

8. Hokkanen, L., Väänänen-Vainio-Mattila, K.: UX work in startups: current practices and future needs. In: Lassenius, C., Dingsøyr, T., Paasivaara, M. (eds.) XP 2015. LNBIP, vol. 212, pp. 81-92. Springer, Heidelberg (2015) 
9. Füller, J., Schroll, R., von Hippel, E.: User generated brands and their contribution to the diffusion of user innovations. Res. Policy 42, 1197-1209 (2013)

10. Sánchez-Gordón, M.-L., O'Connor, R.V.: Understanding the gap between software process practices and actual practice in very small companies. Softw. Qual. J. 1-22 (2015). Online First Articles (ISSN: 0963-9314 (Print) 1573-1367 (Online))

11. ISO: 9241-210:2010. Ergonomics of Human System Interaction-Part 210: Human-Centred Design for Interactive Systems. International Standardization Organization (ISO). Switzerland (2009)

12. Hassenzahl, M.: The interplay of beauty, goodness and usability in interactive products. Proc. HCI 19(4), 319-349 (2004). Lawrence Erlbaum Associates

13. Väänänen-Vainio-Mattila, K., Roto, V., Hassenzahl, M.: Towards practical user experience evaluation methods. EL-C. In: Meaningful Measures: Valid Useful User Experience Measurement (VUUM), pp. 19-22 (2008)

14. May, B.: Applying lean startup: an experience report: lessons learned in creating \& launching a complex consumer app. In: Agile Conference (AGILE), pp. 141-147. IEEE (2012)

15. Klein, L.: UX for Lean Startups: Faster Smarter User Experience Research and Design. O'Reilly Media Inc, Newton (2013)

16. Guba, E.G.: Criteria for assessing the trustworthiness of naturalistic inquiries. ECTJ 29(2), 75-91 (1981) 